\title{
Structural and mechanistic basis of the high catalytic activity of monooxygenase Tet(X4) on tigecycline
}

Qipeng Cheng ${ }^{1,2}$, Yanchu Cheung ${ }^{2}$, Chenyu Liu², Qingjie Xiao ${ }^{3}$, Bo Sun ${ }^{3}$, Jiahai Zhou ${ }^{4}$, Edward Wai Chi Chan', Rong Zhang ${ }^{5}$ and Sheng Chen ${ }^{2 *}$ (i)

\begin{abstract}
Background: Tigecycline is a tetracycline derivative that constitutes one of the last-resort antibiotics used clinically to treat infections caused by both multiple drug-resistant (MDR) Gram-negative and Gram-positive bacteria. Resistance to this drug is often caused by chromosome-encoding mechanisms including over-expression of efflux pumps and ribosome protection. However, a number of variants of the flavin adenine dinucleotide (FAD)dependent monooxygenase Tet $X$, such as Tet(X4), emerged in recent years as conferring resistance to tigecycline in strains of Enterobacteriaceae, Acinetobacter sp., Pseudomonas sp., and Empedobacter sp. To date, mechanistic details underlying the improvement of catalytic activities of new TetX enzymes are not available.

Results: In this study, we found that Tet(X4) exhibited higher affinity and catalytic efficiency toward tigecycline when compared to Tet $\left(X_{2}\right)$, resulting in the expression of phenotypic tigecycline resistance in $E$. coli strains bearing the tet(X4) gene. Comparison between the structures of Tet(X4) and Tet(X4)-tigecycline complex and those of Tet(X2) showed that they shared an identical FAD-binding site and that the FAD and tigecycline adopted similar conformation in the catalytic pocket. Although the amino acid changes in Tet(X4) are not pivotal residues for FAD binding and substrate recognition, such substitutions caused the refolding of several alpha helixes and beta sheets in the secondary structure of the substrate-binding domain of Tet(X4), resulting in the formation of a larger number of loops in the structure. These changes in turn render the substrate-binding domain of Tet(X4) more flexible and efficient in capturing substrate molecules, thereby improving catalytic efficiency.
\end{abstract}

Conclusions: Our works provide a better understanding of the molecular recognition of tigecycline by the TetX enzymes; these findings can help guide the rational design of the next-generation tetracycline antibiotics that can resist inactivation of the TetX variants.

Keywords: Tet(X4), Variant, Tigecycline, FAD binding, Secondary structure

\footnotetext{
* Correspondence: shechen@cityu.edu.hk

${ }^{2}$ Department of Infectious Diseases and Public Health, Jockey Club College

of Veterinary Medicine and Life Sciences, City University of Hong Kong,

Kowloon, Hong Kong

Full list of author information is available at the end of the article
}

C C The Author(s). 2021 Open Access This article is licensed under a Creative Commons Attribution 4.0 International License, which permits use, sharing, adaptation, distribution and reproduction in any medium or format, as long as you give appropriate credit to the original author(s) and the source, provide a link to the Creative Commons licence, and indicate if changes were made. The images or other third party material in this article are included in the article's Creative Commons licence, unless indicated otherwise in a credit line to the material. If material is not included in the article's Creative Commons licence and your intended use is not permitted by statutory regulation or exceeds the permitted use, you will need to obtain permission directly from the copyright holder. To view a copy of this licence, visit http://creativecommons.org/licenses/by/4.0/ The Creative Commons Public Domain Dedication waiver (http://creativecommons.org/publicdomain/zero/1.0/) applies to the data made available in this article, unless otherwise stated in a credit line to the data. 


\section{Background}

The abusive usage of antibiotics in the past few decades resulted in widespread drug resistance, a clinical and public health problem that poses a significant threat to human health. As a last-resort antibiotic, tigecycline was approved for clinical use by FDA in 2005 and is still effective against multiple-resistant (MDR) pathogens [1, 2]. However, following increased usage of this antibiotic, resistant strains have emerged [3-5]. Previously, tigecycline resistance mechanisms mainly involve activities of non-specific efflux pumps and ribosomal protection [6]. Recently, several plasmid-encoded variants of the tetracycline-degrading enzyme Tet(X), such as Tet(X4) which confers high-level tigecycline resistance, were found to be produced by MDR bacteria isolated from animals and humans [7-12]. These novel enzymes degrade tetracyclines more effectively than Tet $(\mathrm{X})$. Organisms carrying genetic elements that encode these enzymes have been disseminated in clinical and veterinary practices, prompting a public health concern. Despite intensive research to investigate why new TetX variants exhibited higher catalytic activity towards tigecycline, the detailed mechanism involved remains poorly understood.

In this study, we characterized the mechanism underlying the degradation of tigecycline by Tet(X4), resolved the crystal structure of the Tet(X4) and tigecyclineTet(X4) complex, and revealed the substrate basis of Tet(X4)-mediated catalysis. Compared to the structure of Tet(X2), the secondary structure of the substratebinding domain of Tet(X4) deconstructed a large number of $\alpha$-helix and $\beta$-sheet which resulted in the loss of various internal contact points in the structure, rendering the substrate-binding domain more flexible in allowing access of the tigecycline molecule to flavin adenine dinucleotide (FAD) for oxidation.

\section{Results}

\section{Tet(X4) against tetracycline antibiotics}

Consistent with previous studies [8,10,13], E. coli strain BW25113 carrying the plasmid pBAD-18-tet(X4) was found to exhibit an 8-64-fold increase in MIC of various tetracycline antibiotics when compared with the host strain (Table 1). We observed the in vitro degradation of tigecycline by purified recombinant Tet(X4) (Fig. S1), which was characterized by a time-dependent decrease in the 350 420-nm absorbance in the UV absorbance spectrum due to breakage of the conserved $\beta$-diketone chromophore in the tigecycline (Fig. S2) [7].

To explore the substrate binding and catalytic efficiency of Tet(X4), an enzyme kinetic assay was performed on Tet(X4) by continuously monitoring the decrease in UV absorbance at $400 \mathrm{~nm}$ under steady-state conditions. We also purified Tet(X2) and used it as a control by determining the kinetic parameters of Tet(X2) toward tigecycline.
Table 1 Susceptibility of tetracyclines in E. coli BW25113 harboring a pBAD18 vector which contains the tet $(X 2)$, tet $(X 4)$, or mutated tet(X2) gene

\begin{tabular}{llll}
\hline $\begin{array}{l}\text { E. coli } \\
\text { BW25113 } \\
\text { strains }\end{array}$ & MIC $(\mathbf{m g} / \mathbf{L})$ & & \\
\cline { 2 - 4 } & Tetracycline & Minocycline & Tigecycline \\
\hline Vector control & 2 & 2 & 0.25 \\
29522 & 1 & 0.25 & 0.125 \\
Tet $(X 2)$ & 32 & 4 & 1 \\
Tet $(X 4)$ & 64 & 16 & 16 \\
$L^{282} S$ & 32 & 8 & 4 \\
$V^{329} M$ & 32 & 8 & 2 \\
\hline
\end{tabular}

The tigecycline-degrading efficiency of Tet(X4) was about 4.8 folds higher than that of $\operatorname{Tet}(\mathrm{X} 2)$, with the $k_{\mathrm{cat}} / K_{M}$ values being $1.13 \times 10^{6} \mathrm{M}^{-1} \mathrm{~s}^{-1}$ and $2.33 \times 10^{5} \mathrm{M}^{-1} \mathrm{~s}^{-1}$, respectively (Table 2). This difference in catalytic efficiency is due to both increase in substrate turnover $\left(k_{\text {cat }}\right)$ and higher substrate-binding affinity $\left(K_{M}\right)$ by Tet $(\mathrm{X} 4)$ when compared to Tet(X2). The increased activity of Tet(X4) toward tigecycline is similar to that of the previously reported tigecycline resistance-conferring enzyme Tet(X7) [7]. The inactivation of tigecycline by Tet(X4) was also analyzed by ESI-mass spectrometry; the primary product of tigecycline was observed at peak $m / z 586.4$ in all reactions (Fig. S3). A new product peak at $m / z 602.5$ was detected upon incubation of Tet(X4) with tigecycline for $30 \mathrm{~min}$, which is corresponding to the addition of one oxygen atom to tigecycline $(\mathrm{m} / z$ 586.5), suggesting that Tet(X4) is likely a monooxygenase.

\section{Structure of Tet(X4)}

We solved the X-ray crystal structure of Tet(X4) at a resolution of $1.78 \AA$, with the key dataset and refinement statistics being shown in Table S1. It was found to exhibit a typical folding pattern similar to that of the previously reported tetracycline destructase, with a FAD-binding domain, a substrate-binding domain, and a C-terminal $\alpha$-helix bridging the two domains (Fig. 1). Its structure was shown to be almost identical (root-meansquare deviation [RMSD] of $0.35 \AA$ and $0.38 \AA$, respectively) to that of other members of tetracycline destructase, such as Tet(X2) (PDB: 2XDO, with 96.04\% sequence identity) from Bacteroides thetaiotaomicron

Table 2 Kinetic parameters of Tet(X2), Tet(X4), and enzymes carrying the $\mathrm{L}^{282} \mathrm{~S}$ or $\mathrm{V}^{329} \mathrm{M}$ substitutions on tigecycline

\begin{tabular}{llll}
\hline Protein & $\boldsymbol{k}_{\text {cat }}\left(\mathbf{S}^{-1}\right)$ & $\boldsymbol{K}_{\boldsymbol{M}}(\boldsymbol{\mu M})$ & $\boldsymbol{k}_{\text {cat }} / \boldsymbol{K}_{\boldsymbol{M}}\left(\mathbf{M}^{-\mathbf{1}} \mathbf{S}^{-1}\right)$ \\
\hline Tet $(\mathrm{X} 2)$ & $1.04 \pm 0.01$ & $4.45 \pm 0.13$ & $2.33 \times 10^{5}$ \\
Tet $(\mathrm{X} 4)$ & $2.03 \pm 0.03$ & $1.80 \pm 0.09$ & $1.13 \times 10^{6}$ \\
$\mathrm{~L}^{282} \mathrm{~S}$ & $1.36 \pm 0.05$ & $3.96 \pm 0.38$ & $3.43 \times 10^{5}$ \\
$\mathrm{~V}^{329} \mathrm{M}$ & $3.65 \pm 0.10$ & $4.81 \pm 0.32$ & $7.59 \times 10^{5}$ \\
\hline
\end{tabular}




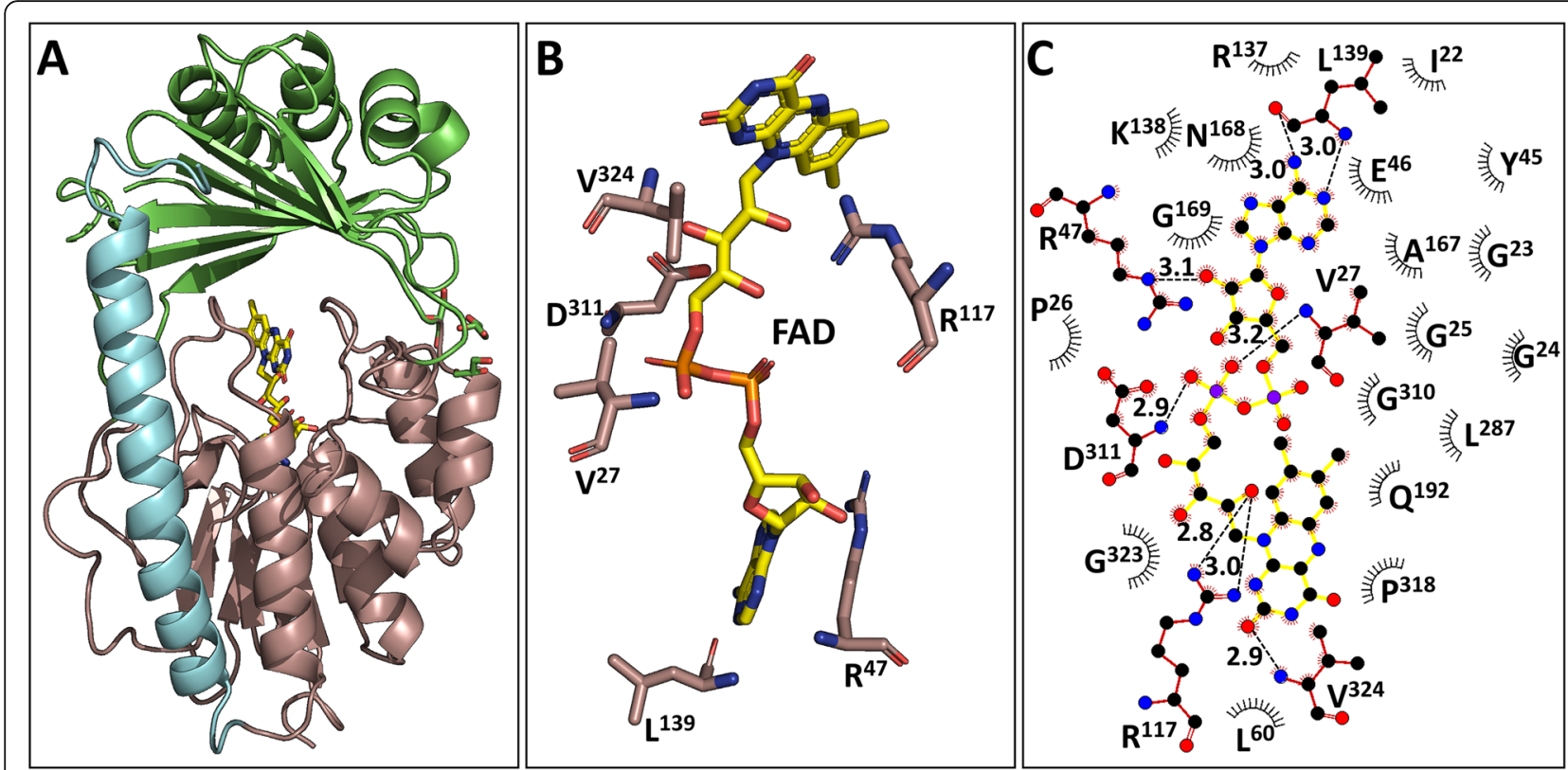

Fig. 1. Crystal structure of Tet(X4). A Overall structure of Tet(X4) has a conserved FAD-binding motif (dark salmon), a substrate-binding domain (green), and a C-terminal bridge helix (cyan). B The FAD-binding site of Tet(X4), FAD is shown in yellow, and protein carbon atoms are depicted in dark salmon. $\mathbf{C}$ Interaction between FAD and Tet(X4). FAD is shown in yellow. Relevant hydrogen bonds are shown as dashed lines and their distances are expressed in $\AA$. The figure was generated by LigPlot+ [14]

and Tet(X7) (PDB: 6WG9, with $88.89 \%$ sequence identity) from Pseudomonas aeruginosa. Only one monomer was observed in the crystallographic asymmetric unit in all structures. The monomer includes 369 amino acids of Tet(X4) $\left(\mathrm{Asn}^{247}, \mathrm{Gln}^{248}\right.$, and $\mathrm{Thr}^{249}$ were not modeled), namely, $\operatorname{Asn}^{12}$ to $\operatorname{Gln}^{383}$. The final $\mathrm{R}$ factor and $\mathrm{R}$ free values of the refined structures varied from 16.63 to $18.53 \%$ and 19.97 to $23.88 \%$, respectively.

In the Tet(X4) and Tet(X4)-tigecycline structures, FAD is bound non-covalently to an IN-conformation such as those of Tet(X) and Tet(X7) (Figs. 1 and 3) [7, 15]. The FAD-binding residues $\left(\mathrm{Val}^{27}, \mathrm{Glu}^{46}, \mathrm{Arg}^{47}, \mathrm{Gly}^{57}, \mathrm{Gly}^{58}\right.$, $\mathrm{Arg}^{117}, \mathrm{Leu}^{139}, \mathrm{Asp}^{311}, \mathrm{Pro}^{318}$, and $\mathrm{Val}^{324}$ ) are conserved in Tet(X2) and Tet(X4). Moreover, multiple sequence alignment (MSA) revealed that these residues are also conserved among all Tet(X) variants (Fig. 2).

\section{Recognition of tigecycline by Tet(X4)}

Accommodation of tigecycline in the binding pocket of Tet(X4) was investigated by X-ray diffraction studies of tigecycline-soaked Tet(X4) crystals. Analysis of the Tet(X4)-tigecycline complex structure revealed specific interactions between the tigecycline hydroxylation product $(\mathrm{T} 1 \mathrm{C})$ and the isoalloxazine of FAD in the large active site cavity (Fig. 3A). Electron density maps allow for unambiguous identification and placement of a $\mathrm{T} 1 \mathrm{C}$ molecule in the crystal. In the Tet(X4)-tigecycline complex structure, the FAD cofactor also adopts the IN-conformation analogous to the Tet $(\mathrm{X} 4)$ structure (Fig. 3A).

The A ring of $\mathrm{T} 1 \mathrm{C}$ forms hydrogen bonds with the side chain of $\operatorname{Gln}^{192}$ (Fig. 3B, C). A water molecule (Wat ${ }^{9}$ ) establishes a hydrogen-bonding bridge connecting the hydroxyl group of $\mathrm{Ser}^{238}$ to the carbonyl O atom of $\mathrm{Gln}^{192}$; this type of interaction was also observed in the TetX-minocycline complex structure [18]. Like the previously reported Tet(X)tetracycline complex structures $[15,18,19]$, the cofactor FAD in the deep cavity of Tet(X4) was also found to form hydrogen bonds with the hydroxyl groups at C1c and C12 of $\mathrm{T} 1 \mathrm{C}$ via the $\mathrm{N}^{5}$ and $\mathrm{O}^{4}$ atoms (Fig. $3 \mathrm{~B}, \mathrm{C}$ ), which plays a key role in stabilizing substrate binding. Another water molecule (Wat ${ }^{64}$ ) also forms a hydrogen bond (3.2 $\AA$ ) with the hydrophilic region (C21) of T1C (Fig. 3B, C). The hydrophilic sites $\mathrm{Arg}^{213}, \mathrm{Ala}^{225}, \mathrm{H}^{234}, \mathrm{Ala}^{320}, \mathrm{G}^{321}, \mathrm{Glu}^{367}$, and $\mathrm{Asn}^{371}$ were found to be able to interact (3.0-4.0 $\AA$ ) with the hydroxyl sites at C11, C21, C10, and C91 of T1C. Since T1C does not have any specific group at $\mathrm{C} 5, \mathrm{C} 6, \mathrm{C} 8$, and $\mathrm{C} 9$, only the functional group of $\mathrm{C} 7$ makes van der Waals contacts with the side chains of $\mathrm{Met}^{215}$, $\mathrm{Asn}^{371}$, and $\mathrm{Met}^{375}$ (within $4.0 \AA$ A). Furthermore, the hydrophobic segment of T1C (C41-C7) forms several interactions with the side chains of hydrophobic residues $\mathrm{Phe}^{224}, \mathrm{Pro}^{318}$, and $\mathrm{Phe}^{319}$. These observations indicate that recognition of tigecycline by the active site of Tet(X4) mainly involves targeting of the conserved hydrophilic substituents in the A ring and $\mathrm{C} 10, \mathrm{C} 11$, and $\mathrm{C} 12$ of $\mathrm{T} 1 \mathrm{C}$. The Tet(X4)-tigecycline complex structure is highly identical to the Tet(X2)-tigecycline complex structure, with RMSD at 0.4 


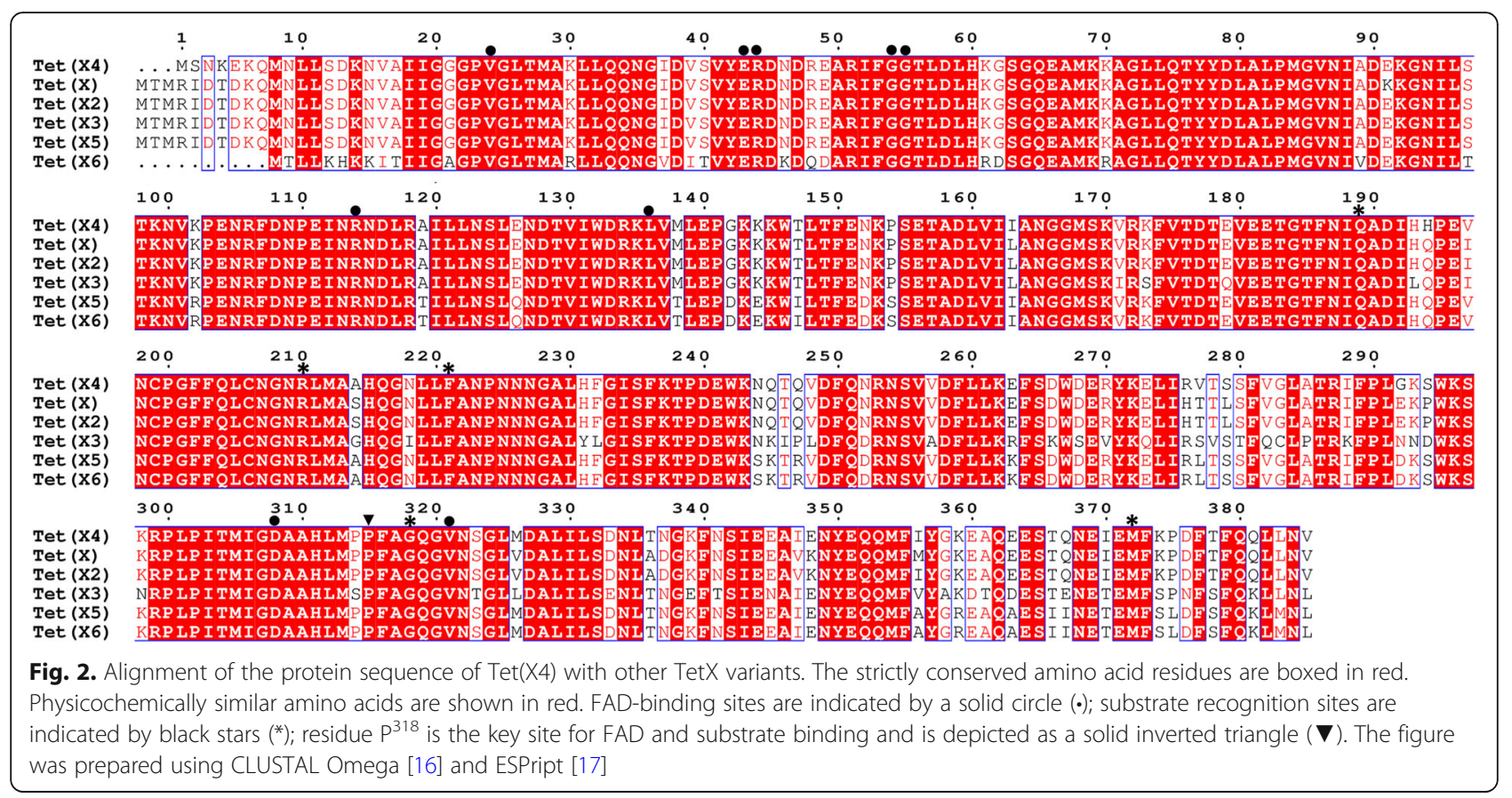

A. T1C adopts similar conformations that interact with $\mathrm{Gln}^{192}, \mathrm{Arg}^{213}, \mathrm{Phe}^{224}, \mathrm{Pro}^{318}, \mathrm{Gly}^{321}$, and $\mathrm{Met}^{375}$, which is observable in both the Tet(X4)-tigecycline and Tet(X2)-tigecycline complex structures (Fig. 4). Such features are also detectable in other tetracycline-antibiotic complex structures $[15,18,19]$.

The RMSD of the $C \beta$ positions between the tigecycline complex structure and $\operatorname{Tet}(\mathrm{X} 4)$ is $0.1 \AA$, suggesting that insertion of the tigecycline molecule did not cause dramatic conformational changes in the Tet(X4) monooxygenase structure (Fig. 4A). Upon capturing of tigecycline by Tet(X4), the loop between $\alpha 10$ and $\alpha 11$ exhibited a dynamic shift in position when compared to the structure of $\mathrm{Tet}(\mathrm{X} 4)$, with $\mathrm{C} \alpha$ of $\mathrm{Ala}^{320}$ and $\mathrm{Gln}^{322}$ being shifted $1.3 \AA$ and $1.5 \AA$, respectively (Figs. 4A and 5). These changes might expand the cavity and facilitate contact with FAD. Furthermore, when compared to Tet(X4) structure, the value of the B-factor of the loop $\left(\mathrm{H}^{314}-\mathrm{G}^{323}\right)$ was found to have increased from 22.6 to $52.9 \AA^{2}$ in the Tet(X4)-tigecycline complex structure, indicating that the loop exhibits higher mobility for ligand binding and release. This observation also shows that the loop $\left(\mathrm{H}^{314}-\mathrm{G}^{323}\right)$ plays an important role in substrate recognition by Tet(X4).
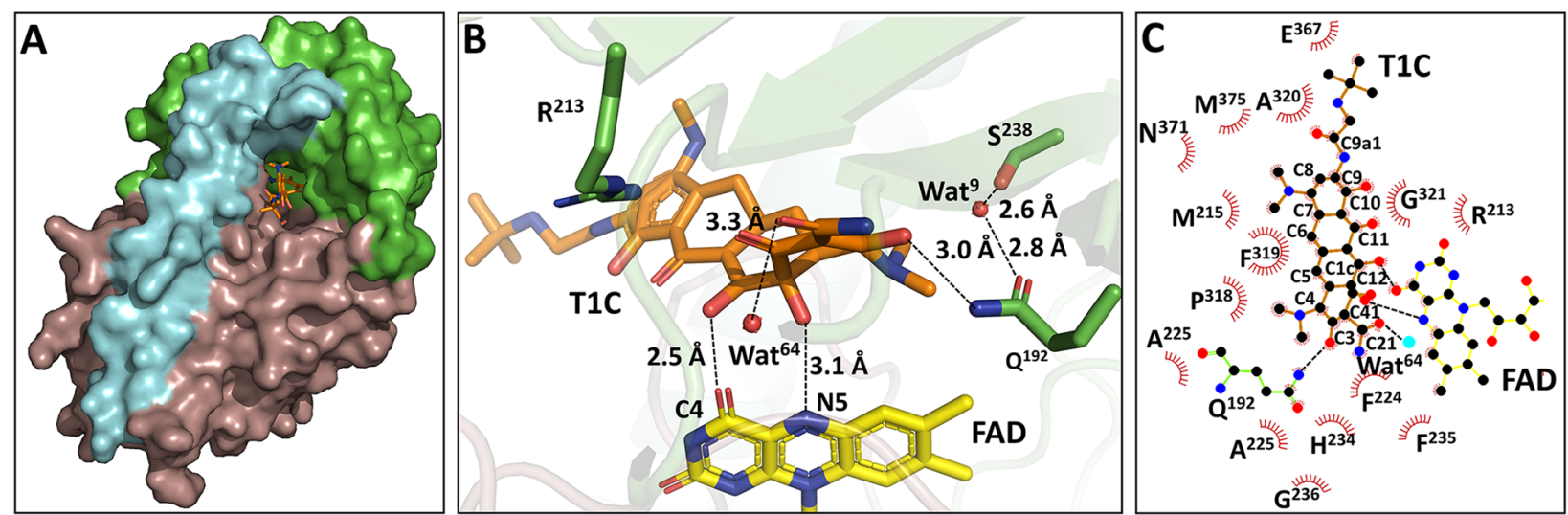

Fig. 3. Overview of the process by which tigecycline binds to Tet(X4). A Surface representation of the tigecycline (T1C) binding cavity. B Active site recognition of $\mathrm{T} 1 \mathrm{C}$ by hydrogen-bonding of $\mathrm{Tet}(\mathrm{X} 4)$ (protein residues in green, $\mathrm{T} 1 \mathrm{C}$ in orange, FAD in yellow, hydrogen bonds as black dashed lines). $\mathbf{C}$ Interaction between T1C and Tet(X4). Residues around the binding pocket are depicted as green sticks, T1C is depicted in orange, and FAD is depicted in yellow 

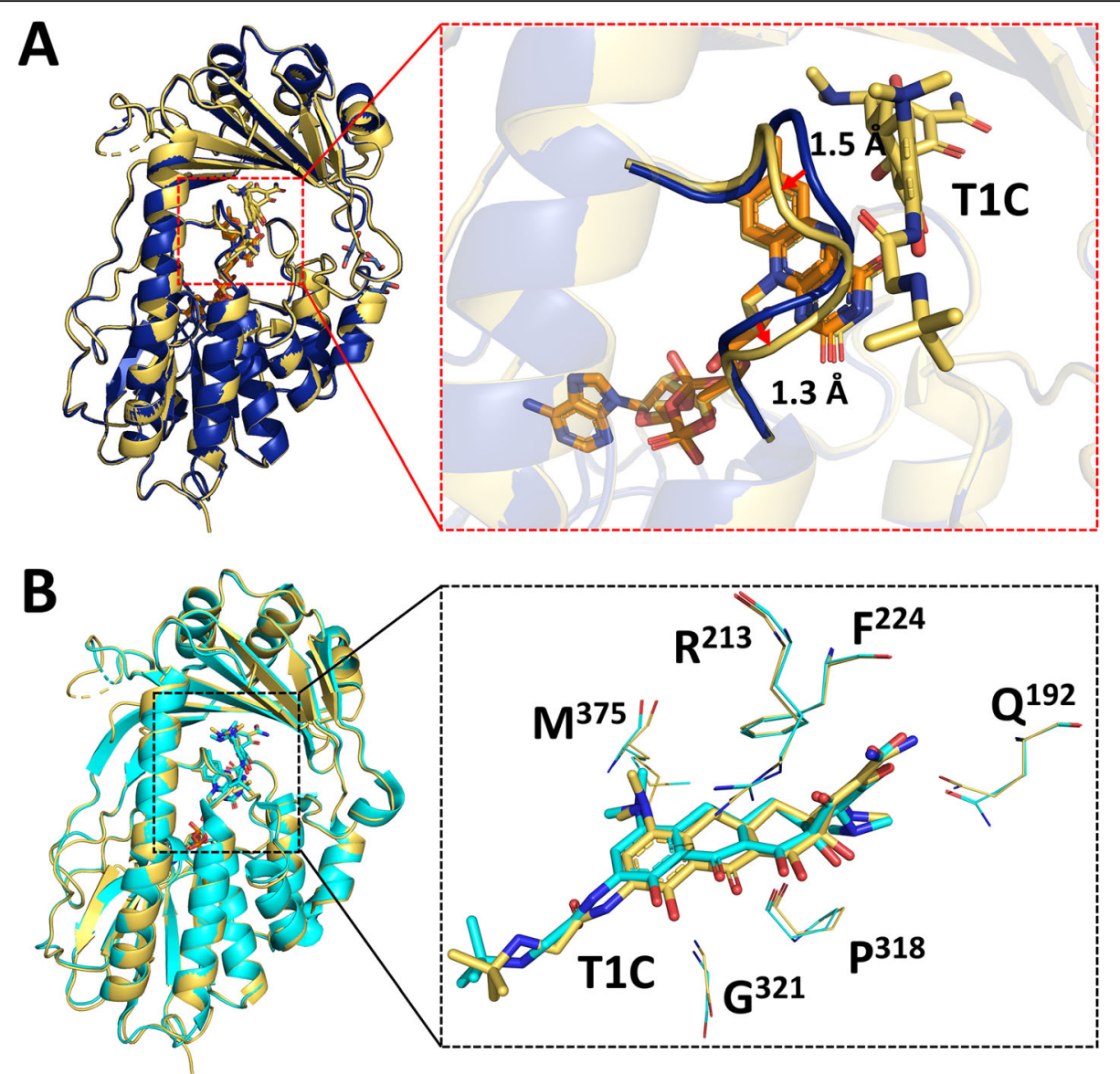

Fig. 4. Structural comparison of Tet(X4)-tigecycline complex with Tet(X4) and Tet(X2)-tigecycline complex. A Cartoon and superimposition of Tet(X4)-tigecycline structure (the color of the cartoon is shown in yellow) and Tet(X4) structure (the color of the cartoon is shown in blue). The red dashed box indicates the dynamic changes in the loop between a10 and a11, with details shown in the right enlarged dashed box. FAD and T1C are shown as sticks. B Superposition of the structure of Tet(X4)-tigecycline complex (the color of the cartoon is shown in yellow) with the Tet(X2)-tigecycline complex (4A6N, the color of the cartoon is shown in cyan). The black dashed box indicates the interactions of TIC with Tet(X4) and Tet(X2), with details shown in the right enlarged dashed box. T1C are shown as sticks; conserved interacting residues are depicted as lines

\section{Secondary structure changes in the substrate-binding domain of Tet(X4)}

Superimposition of the structure of Tet(X4) and structure of Tet(X2) revealed the difference between the substrate-binding domain of the two enzymes (Fig. 5). First, $\alpha 10$ and $\alpha 11$ in the Tet(X4) structure were shorter when compared to Tet(X2), indicating that the loop between $\alpha 10$ and $\alpha 11$ was extended to allow accommodation of a more flexible conformation. The loop has been considered the substratebinding site; hence, an extended loop might enhance the chance by which tigecycline attaches to FAD for oxidization. In addition, a number of alpha helixes in Tet(X4), namely $\alpha 6, \alpha 7, \alpha 8$, and $\alpha 9$, have collapsed to generate more loops for substrate binding (Fig. 5). $\alpha 4$ formed an alpha helix structure in the Tet(X2) structure, but it was deconstructed as a loop in Tet(X4) structure (Fig. 5). As the main components of the substrate-binding domain, $\beta 12$ and $\beta 16$ in Tet(X4) have also become smaller in size, forming a larger number of loops when compared to Tet(X2) (Fig. 5). These structural alterations allowed the secondary structure of $\mathrm{Tet}(\mathrm{X} 4)$ to adopt more loops and turns, rendering the structure of the substrate-binding domain more flexible and more readily to accommodate the tetracycline molecule, resulting in increased catalytic efficiency of enzyme on tigecycline. These structural features therefore explain the phenotype resistance of Tet(X4)-producing organisms.

The changes in the secondary structure of Tet(X4) have been supported by CD scan and FTIR analysis (Table 3, Figs. S4 and S5). CD spectroscopic studies revealed that the composition of $\alpha$-helix (29.3\%) and $\beta$-sheet (24.7\%) of Tet(X4) have slightly decreased by $2-3 \%$ when compared to Tet(X2). FTIR analysis of Tet(X4) protein solution, which indicated 

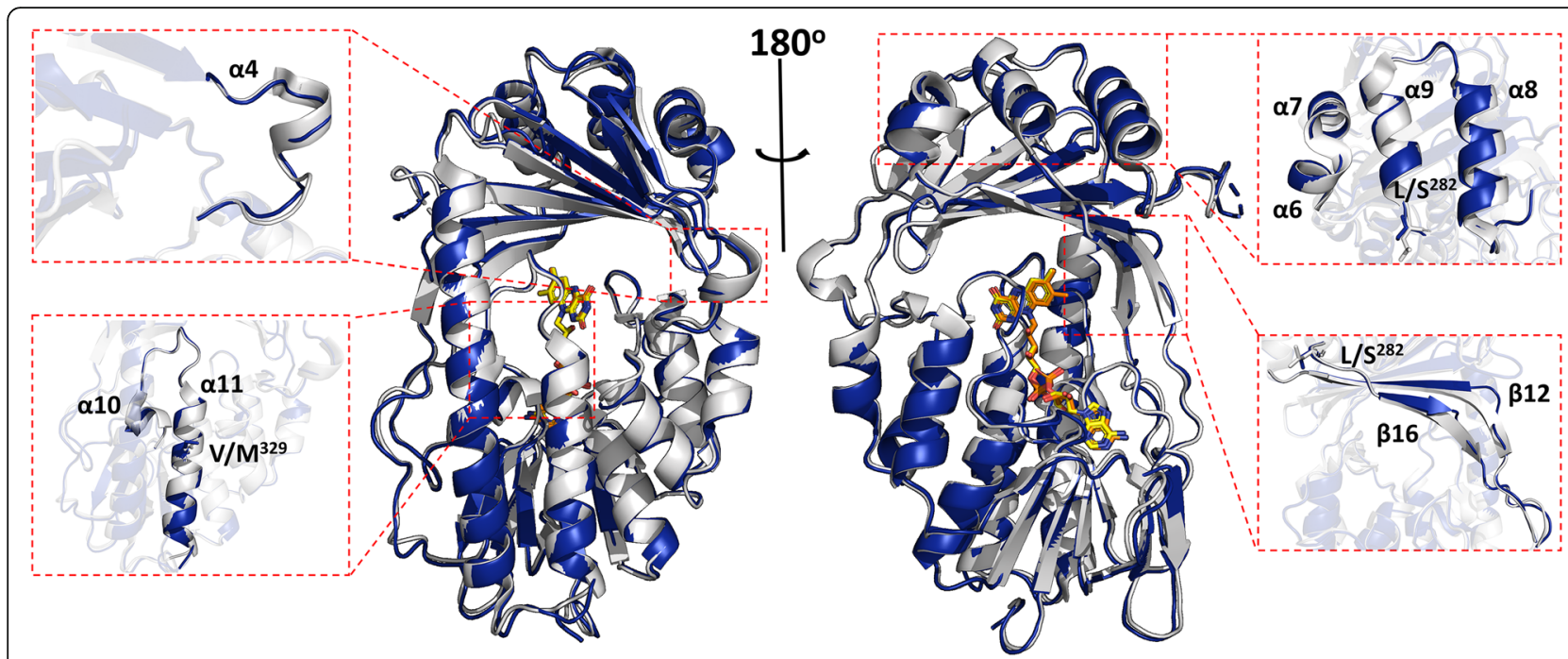

Fig. 5. Superimposition of the Tet( $X 4)$ structure (cartoon of Tet $(X 4)$ shown in blue) and Tet( $X 2)$ structure (cartoon of Tet( $X 2)$ shown in gray). FAD in Tet $(X 4)$ is shown in orange, FAD in Tet(X2) is shown in yellow; the red dashed box indicates the structural difference between the substratebinding domain of $\operatorname{Tet}\left(X_{4}\right)$ and $\operatorname{Tet}\left(X_{2}\right)$, with details shown in the enlarged dashed box; key residues of Tet( $\left.X 4\right)$ that are different from Tet( $\left.X 2\right)$ are depicted as sticks

that the proportion of helices ( $\alpha$-helix and $3_{10}$-helix) and $\beta$ sheet declined to $30.8 \%$ and $40.4 \%$, respectively (Table 3 ), is consistent with the results of CD experiments. All in all, it was evident that the percentage of $\alpha$-helix and $\beta$-sheet has decreased significantly in Tet(X4), rendering the secondary structure of Tet(X4) more flexible.

Amino acid substitutions cause changes in the secondary structure of Tet(X4)

Compared to Tet(X2), the variant residues in $\operatorname{Tet}(\mathrm{X} 4)$ are not in vital sites for FAD binding and substrate recognition, but such amino acid changes might drastically affect the secondary structure of Tet(X4). To confirm our hypothesis, we introduced $\mathrm{L}^{282} \mathrm{~S}$ or $\mathrm{V}^{329} \mathrm{M}$ into Tet(X2) by site-directed mutagenesis. Residues 282 connected $\alpha 9$ and $\beta 16$ (Fig. 5); when the amino acid leucine was substituted by serine at position 282, analyses by CD and FTIR both showed that the percentage of $\alpha$-helix and $\beta$-sheet in the $\mathrm{L}^{282} \mathrm{~S}$-bearing protein decreased significantly, indicating that the $\mathrm{L}^{282} \mathrm{~S}$ substitution caused a drastic change in the secondary structure of the protein (Figs. S4 and S5, Table 3). In contrast, FTIR analysis showed that the proportion of $\beta$ sheet remained steady at $50.7 \%$ in the $\mathrm{V}^{329} \mathrm{M}$-bearing variant enzyme, but the percentage of $\alpha$ helices decreased to 26.3\% (Fig. S4, S5, Table 3). As residue 329 is located at $\alpha 11$ (Fig. 5), the amino acid substitution $\mathrm{V}^{329} \mathrm{M}$ might extend the loop between $\alpha 10$ and $\alpha 11$ but has less effect on $\beta$-sheet. Furthermore, E. coli BW25113 carrying the $\mathrm{L}^{282} \mathrm{~S}$ or $\mathrm{V}^{329} \mathrm{M}$ changes exhibited a 2-4-fold increase in MIC when compared to the Tet(X2)-producing strain. Consistently, the tigecycline-catalyzing efficiency of $\mathrm{L}^{282} \mathrm{~S}$ and $\mathrm{V}^{329} \mathrm{M}$ also increased $1.5-3.5$ folds when compared to Tet(X2). These findings indicate that amino acid substitutions could cause changes in the secondary structures of Tet(X4) and improve the catalytic activity of the enzyme.

\section{Discussion}

Since their discovery in the 1940s, tetracyclines have become the key antimicrobial agents in agricultural, veterinary, and clinical applications $[20,21]$. As a

Table 3 Comparison of the relative content (\%) of different types of secondary structure in Tet(X4), Tet(X2), and enzymes harboring the $\mathrm{L}^{282} \mathrm{~S}$ and $\mathrm{V}^{329} \mathrm{M}$ substitution

\begin{tabular}{|c|c|c|c|c|c|c|c|c|}
\hline & \multicolumn{4}{|c|}{ CD analysis } & \multicolumn{4}{|c|}{ FTIR analysis } \\
\hline & $\overline{\operatorname{Tet}(X 2)}$ & Tet(X4) & $\mathrm{L}^{282} \mathrm{~S}$ & $\mathrm{~V}^{329} \mathrm{M}$ & $\overline{T e t}(X 2)$ & Tet(X4) & $\mathrm{L}^{282} \mathrm{~S}$ & $\mathrm{~V}^{329} \mathrm{M}$ \\
\hline a-helix (\%) & 32.4 & 29.3 & 31.6 & 26.9 & 17.1 & 16.4 & 15.5 & 14.9 \\
\hline $3_{10}$-helix (\%) & - & - & - & - & 26.7 & 14.4 & 11.8 & 11.4 \\
\hline$\beta$-sheet (\%) & 26.1 & 24.7 & 23.9 & 23.9 & 50 & 40.4 & 43.8 & 50.7 \\
\hline Others (\%) & 41.5 & 46 & 44.5 & 49.2 & 6.8 & 28.8 & 28.9 & 23 \\
\hline Spectral deviation (RMSD) & 0.182 & 0.132 & 0.123 & 0.109 & - & - & - & - \\
\hline
\end{tabular}


result, resistance to tetracycline antibiotics became increasingly observed in both Gram-negative and Grampositive bacteria. Such resistance phenotypes were found to be caused by efflux activities and ribosome protection [21, 22]. Subsequent advancements in tetracycline modification techniques resulted in the third- and fourth-generation tetracyclines such as tigecycline, eravacycline, and omadacycline, which could effectively combat tetracycline resistance [23-25]. However, the newly discovered plasmid-borne tet $(\mathrm{X})$ genes, which encode tetracycline inactivation enzymes such as TetX4, were found to be responsible for conferring resistance to the latest-generation tetracycline antibiotics among MDR Gram-negative pathogens $[8,10]$. Furthermore, with increasing selective pressure imposed by the last generation tetracyclines, organisms that carry novel tet $(\mathrm{X})$ variants, such as tet(X5-14), have emerged and disseminated extensively $[7,9,11,26]$. However, the structural basis of the increased catalytic activity of Tet(X4) remains unknown.

In this work, we showed that Tet(X4) exhibited a high affinity toward tigecycline and catalyzed tigecycline more efficiently than Tet(X2). These functional properties of Tet(X4) are responsible for causing the phenotype of tigecycline resistance in $E$. coli strains bearing the tet $(\mathrm{X} 4)$ gene. Interestingly, Tet(X4) shared a highly identical FAD-binding site with that of $\operatorname{Tet}(\mathrm{X} 2)$, with tigecycline being accommodated in a similar conformation in the catalytic cavity. Compared to Tet(X2), the amino acid changes in Tet(X4) are not the pivotal residues for FADbinding and substrate recognition. However, these amino acid substitutions, such as $\mathrm{L}^{282} \mathrm{~S}$ and $\mathrm{V}^{329} \mathrm{M}$, might cause the refolding of several alpha helixes and beta sheets in the secondary structure of the substratebinding domain of Tet(X4), allowing a larger number of loops to form in the structure. These changes render the substrate-binding domain of Tet(X4) more flexible and efficient in capturing substrates and thereby improving catalysis. Loop regions as the most flexible parts of protein structures often play an important role in protein functions by interacting with the solvent and substrates [27]. The finding that the dynamic change of structure of TetX4 resulted in an enhanced catalytic activity is consistent with that of a previous report on the effect of directed evolution of Tet(X) toward tigecycline [28].

Structure-guided approaches have been proven to be effective in assisting the discovery of antibiotic analogs and inhibitors $[29,30]$. The high-resolution structures of Tet(X4) and Tet(X4)-tigecycline complex provided a deep understanding of the molecular recognition mechanism of tigecycline by Tet(X)s enzymes; such knowledge facilitates rational design of novel tetracycline antibiotics that can escape enzymatic inactivation and remain active against a wide range of MDR bacterial pathogens.

\section{Conclusions}

In conclusion, we investigated the structural basis of the high catalytic activity of Tet(X4) on tigecycline, which is one of the last line antibiotics used to treat multidrugresistant bacterial infections. We found that Tet(X4) exhibited a high affinity toward tigecycline and catalyzed tigecycline more efficiently than Tet(X2). These functional properties were due to carriage of specific amino acid substitutions, such as $\mathrm{L}^{282} \mathrm{~S}$ and $\mathrm{V}^{329} \mathrm{M}$, in Tet(X4) which might cause the refolding of several alpha helixes and beta sheets in the secondary structure of the substrate-binding domain, allowing a larger number of loops to form in the structure. These changes render the substrate-binding domain of Tet(X4) more flexible and efficient in capturing substrates and thereby improving catalysis. These findings facilitate the design of nextgeneration tetracycline which can resist degradation by Tet(X4).

\section{Methods}

\section{Bacterial strains and functional cloning of tet $(X) s$}

The full-length tet $(\mathrm{X} 4)$ gene was amplified from genomic DNA of ST767 E. coli strain [13] by two pairs of primers (Table S2). The PCR product which contained the EcoR I / Sal I restriction sites was sub-cloned into the plasmid vector pBAD18-kan (kanamycin resistance), which contained the arabinose $\mathrm{pBAD}$ promoter. Another tet $(\mathrm{X} 4)$ gene PCR product with the $B a m H$ I/Xho I restriction sites was ligated to a modified $\mathrm{pET}-\mathrm{M}$ vector which contained $3 \mathrm{C}$ protease cleave site beyond the His 6 tag. Subsequently, the recombinant plasmid pBAD-18-tet $(\mathrm{X} 4)$ was transformed into competent cells of E. coli BW25113, followed by antibiotic susceptibility tests. The recombinant plasmid pET-M-tet (X4) was transformed into competent $E$. coli BL21 (DE3) cells for protein expression and purification. We also constructed two tet $(\mathrm{X} 2)$-bearing vectors as control. Mutations were introduced into the tet $(\mathrm{X} 2)$ gene using the QuickChange SiteDirected Mutagenesis Kit (Stratagene).

\section{Antimicrobial susceptibility test}

Antibiotic susceptibility test of E. coli strain BW25113 harboring the tet $(\mathrm{X} 4)$-bearing $\mathrm{pBAD} 18$-kan vector was performed. Minimum inhibitory concentrations (MICs) were determined according to Clinical and Laboratory Standards Institute (CLSI) procedures using MuellerHinton broth microdilution method [31]. E. coli strain ATCC 25922 was used as quality control.

\section{Protein expression and purification}

0.5 liter of Luria Broth (LB) containing $100 \mu \mathrm{g} / \mathrm{mL}$ ampicillin was inoculated into a $5-\mathrm{mL}$ overnight culture, followed by incubation with shaking at $37{ }^{\circ} \mathrm{C}$ until an optical density of 0.6 at $600 \mathrm{~nm}$ (OD600) was reached. 
The expression of enzymes was induced by $0.5 \mathrm{mM}$ isopropyl- $\beta$-D-thiogalactopyranoside (IPTG) at $16^{\circ} \mathrm{C}$ for $16 \mathrm{~h}$. The cells were harvested by centrifugation at $11,300 \times g$ for $5 \mathrm{~min}$ and resuspended in lysis buffer ( 25 $\mathrm{mM}$ Tris- $\mathrm{HCl}, \mathrm{pH} 8.0,300 \mathrm{mM} \mathrm{NaCl}, 30 \mathrm{mM}$ imidazole, and $1 \mathrm{mM}$ PMSF) and then broken by high-pressure homogenization. The soluble fractions were passed through a Ni-nitrilotriacetic acid (NTA) column, rinsed with $25 \mathrm{mM}$ Tris- $\mathrm{HCl}, \mathrm{pH} 8.0,300 \mathrm{mM} \mathrm{NaCl}$, and 30 $\mathrm{mM}$ imidazole, and finally eluted with $25 \mathrm{mM}$ Tris- $\mathrm{HCl}$, $\mathrm{pH}$ 8.0, $300 \mathrm{mM} \mathrm{NaCl}$, and $300 \mathrm{mM}$ imidazole. The eluted proteins were concentrated using the Amicon Ultra-15 (nominal molecular weight limit [NMWL] $=30$ 000) centrifugal filter device. The purified enzyme was incubated with $3 \mathrm{C}$ protease at $4{ }^{\circ} \mathrm{C}$ overnight to remove the His6 tag. The target proteins were further purified by gel filtration chromatography (Superdex 75; GE Healthcare) in a buffer of $20 \mathrm{mM}$ Tris (pH 7.5), $150 \mathrm{mM}$ $\mathrm{NaCl}$, and $2 \mathrm{mM}$ DTT (Dithiothreitol). The desired fractions were collected and concentrated. The purity of the protein was determined by sodium dodecyl sulfatepolyacrylamide gel electrophoresis (SDS-PAGE) (Fig. S1).

\section{Steady-state kinetics of Tet(X4)}

Each $500-\mu \mathrm{L}$ reaction was prepared with $100 \mathrm{mM}$ TAPS buffer at $\mathrm{pH} 8.5$ with $0-50 \mu \mathrm{M}$ substrate, $5 \mathrm{mM} \mathrm{MgCl}$, and $0.5 \mathrm{mM}$ nicotinamide adenine dinucleotide phosphate (NADPH). UV-visible spectroscopy measurements were performed in triplicate at 400-nm wavelength light, using a UV-1900 UV-Vis spectrophotometer (Shimadzu) for measurement for $3 \mathrm{~min}$ at room temperature. Initial reaction velocities were determined by linear regression using the UVProbe 2.70 Software and fitted to the Michaelis-Menten equation by GraphPad Prism 8.

\section{Crystallization, data collection, and structure refinement}

Tet(X4) protein was concentrated to $22 \mathrm{mg} / \mathrm{mL}$ and crystallized by sitting drop vapor diffusion at $16{ }^{\circ} \mathrm{C}$ in $0.2 \mathrm{M}$ ammonium acetate, $0.1 \mathrm{M}$ sodium citrate tribasic dihydrate $\mathrm{pH} 5.6$, and $30 \%(\mathrm{w} / \mathrm{v})$ polyethylene glycol 4000. Tigecycline was soaked into the crystals by incubating the Tet(X4) crystals in a reservoir buffer for 30 min. The crystals were cryoprotected by $25 \%$ glycerol in reservoir buffer for $10 \mathrm{~s}$ and flash-cooled in liquid nitrogen. Diffraction data were collected at $100 \mathrm{~K}$ on beamline BL17U1 at the Shanghai Synchrotron Radiation Facility [32]. The diffraction data were processed by $x d s$ [33], xia2 [34], and aimless [35]. The Tet(X4) structure was solved by molecular replacement using Phaser, with free Tet(X) structure (PDB: 2XYO) as the search model. Structure refinement was performed by using Phenix [36], REFMAC [37], and Coot [38]. The structures have been deposited to PDB as 7EPV and 7EPW (validated by
PDB, Additional file 8). The structure figures were prepared by PyMOL [39].

\section{Fourier transform infrared (FTIR) spectroscopy}

Infrared spectra were recorded on a PerkinElmer Spectrum 100 instrument using an attenuated total reflection (ATR) sampling accessory as described in previous studies [40, 41]. Briefly, protein solutions were loaded in the well, and data was acquired at $25{ }^{\circ} \mathrm{C}$ in the range of $1700-1600 \mathrm{~cm}^{-1}$ (wavenumber). Typically, eight scans were collected and averaged for a single spectrum with a resolution of $4 \mathrm{~cm}^{1}$. The background was corrected before scanning the samples. The FITR spectra of Tet(X2), Tet(X4), $\mathrm{L}^{282} \mathrm{~S}$, and $\mathrm{V}^{329} \mathrm{M}$ were collected in a buffer of $20 \mathrm{mM}$ Tris $(\mathrm{pH} 7.5)$ and $150 \mathrm{mM} \mathrm{NaCl}$; the absorbance of the buffer was subtracted from the spectra of Tet(X2), Tet(X4), $\mathrm{L}^{282} \mathrm{~S}$, and $\mathrm{V}^{329} \mathrm{M}$. The concentrations of Tet(X2), Tet(X4), $\mathrm{L}^{282} \mathrm{~S}$, and $\mathrm{V}^{329} \mathrm{M}$ in the assay were $20-30 \mathrm{mg} / \mathrm{mL}$.

\section{Circular dichroism (CD)}

CD spectra were measured by a Jasco J-1500 spectropolarimeter at $25{ }^{\circ} \mathrm{C}$. $\mathrm{CD}$ measurements in a spectral range of 260 to $200 \mathrm{~nm}$ were performed, with an interval of 1 $\mathrm{nm}$ and scanning speed of $50 \mathrm{~nm}$ min $^{-1}$ corrected with baseline. The CD spectra of Tet(X2), Tet(X4), $\mathrm{L}^{282} \mathrm{~S}$, and $\mathrm{V}^{329} \mathrm{M}$ protein solutions were acquired, and changes in $\mathrm{CD}$ spectral results were analyzed. The secondary structures were determined by BeStSel [42, 43].

\section{Abbreviations}

ATR: Attenuated total reflection; CD: Circular dichroism; CLSI: Clinical and Laboratory Standards Institute; DTT: Dithiothreitol; FAD: Flavin adenine dinucleotide; FTIR: Fourier transform infrared; IPTG: Isopropyl- $\beta$-Dthiogalactopyranoside; LB: Luria Broth; MDR: Multiple drug resistant; MICS: Minimum inhibitory concentrations; NADPH: Nicotinamide adenine dinucleotide phosphate; NTA: Ni-nitrilotriacetic acid; RMSD: Root-meansquare deviation; SDS-PAGE: Sodium dodecyl sulfate-polyacrylamide gel electrophoresis

\section{Supplementary Information}

The online version contains supplementary material available at https://doi org/10.1186/s12915-021-01199-7.

Additional file 1: Figure S1. Size-exclusion chromatogram of Tet(X4), peak 2 is corresponded to Tet(X4); the inset shows reduced SDS-PAGE analysis of the purified proteins.

Additional file 2: Figure S2. (A) Chemical structure of tigecycline. Rings $B-D$ is responsible for $\beta$-diketone chromophore that was circled by the orange rectangle. (B) In vitro absorbance scan at wavelength between $300 \mathrm{~nm}$ and $500 \mathrm{~nm}$ taken at 60 seconds intervals, covering the Tet(X4) protein, $\mathrm{NADPH}, \mathrm{MgCl}_{2}$, and tigecycline. The rainbow shape illustrates the spectral change over time. Time-dependent decrease in absorbance from $370 \mathrm{~nm}$ to $420 \mathrm{~nm}$ indicates enzymatic disruption of the characteristic tigecycline $\beta$-diketone chromophore and consumption of NADPH.

Additional file 3: Figure S3. Mass spectrometry analysis of enzymatic reactions with tigecycline as substrate. (A), Reaction without enzyme at 0 minutes; (B), Reaction without enzyme at 30 minutes; (C), Reaction of 
Tet(X4) with tigecycline as substrate at 0 minutes; (D), Reaction of Tet(X4) with tigecycline as substrate at $30 \mathrm{~min}$

Additional file 4: Table S1. Crystallographic data and refinement statistics.

Additional file 5: Figure S4. (A) Representative mean spectra of Tet(X2) protein solution shown in the range $1600-1700 \mathrm{~cm}^{-1}$ after baseline correction and vectorial normalization; (B) Curve-fitting analysis of amide I band of Tet(X2) protein solution; (C) Representative mean spectra of Tet(X4) protein solution shown in the range $1600-1700 \mathrm{~cm}^{-1}$ after baseline correction and vectorial normalization; (D) Curve-fitting analysis of amide I band of Tet(X4) ; (E) Representative mean spectra of the $\mathrm{L}^{282} \mathrm{~S}$ mutant protein solution shown in the range $1600-1700 \mathrm{~cm}^{-1}$ after baseline correction and vectorial normalization; (F) Curve-fitting analysis of amide I band of the $\mathrm{L}^{282} \mathrm{~S}$ mutant protein; $(\mathrm{G})$ Representative mean spectra of the $\mathrm{V}^{329} \mathrm{M}$ mutant protein solution shown in the range 1600-1700 $\mathrm{cm}^{-1}$ after baseline correction and vectorial normalization; $(\mathrm{H})$ Curvefitting analysis of amide I band of the $\mathrm{V}^{329} \mathrm{M}$ mutant protein.

Additional file 6: Figure S5. (A) Circular dichroic spectral profiles of Tet(X2) protein solution measured at wavelength between $200 \mathrm{~nm}$ and $260 \mathrm{~nm}$; the raw CD spectrum is shown; (B) Circular dichroic spectral profiles of Tet $(X 4)$ protein solution measured at wavelength between 200 $\mathrm{nm}$ and $260 \mathrm{~nm}$; (C) Circular dichroic spectral profiles of the ${ }^{282} \mathrm{~S}$ mutant protein solution measured at wavelength between $200 \mathrm{~nm}$ and $260 \mathrm{~nm}$; (D) Circular dichroic spectral profiles of the $\mathrm{V}^{329} \mathrm{M}$ mutant protein solution measured at wavelength between $200 \mathrm{~nm}$ and $260 \mathrm{~nm}$.

Additional file 7: Table S2. Primers were used in this study

Additional file 8. Validation reports of the structure of Tet $(X 4)$ and Tet(X4)-tigecycline complex.

\section{Acknowledgements}

We are grateful to the staff of the Shanghai Synchrotron Radiation Facility for their assistance in $\mathrm{X}$-ray data collection. We also thank Dr. Xu Jiangtao for providing the Fourier transform infrared (FTIR) spectroscopy training.

\section{Authors' contributions}

Q.C carried out the project under the guidance of S.C, Q.C, Y.C, and C.L who carried out the protein purification and enzyme kinetics; Q.C and J.Z carried out the protein crystallization; Q.C, Q.X, and B.S carried out the structure's determination and refinement; R.Z performed the analysis of FTIR data; E.W.C carried out the manuscript editing. All authors read and approved the final manuscript.

\section{Authors' information}

Qipeng Cheng mainly contributes to this work.

\section{Funding}

The project was supported by Guangdong Major Project of Basic and Applied Basic Research (2020B0301030005) and NSFC/RGC grant (NSFC-RGC, N_PolyU521/18) from the National Natural Science Fund in China and Research Grant Council of the Government of Hong Kong SAR and Internal grant from City University of Hong Kong (SGP/CityU/9380110).

\section{Availability of data and materials}

All data generated or analyzed during this study are included in this published article and its supplementary information files. The structures in this study have been deposited to PDB as 7EPV and 7EPW.

\section{Declarations}

Ethics approval and consent to participate

Not applicable.

\section{Consent for publication}

Not applicable.

\section{Competing interests}

The authors declare that they have no competing interests.

\section{Author details}

'State Key Lab of Chemical Biology and Drug Discovery, Department of Applied Biology and Chemical Technology, The Hong Kong Polytechnic University, Hung Hom, Kowloon, Hong Kong. ${ }^{2}$ Department of Infectious Diseases and Public Health, Jockey Club College of Veterinary Medicine and Life Sciences, City University of Hong Kong, Kowloon, Hong Kong. ${ }^{3}$ Shanghai Advanced Research Institute, Chinese Academy of Sciences, No.239 Zhangheng Road, Shanghai 201204, China. ${ }^{4}$ CAS Key Laboratory of Quantitative Engineering Biology, Shenzhen Institute of Synthetic Biology, Shenzhen Institute of Advanced Technology, Chinese Academy of Sciences, Shenzhen 518055, China. ${ }^{5}$ Department of Clinical Laboratory, Second Affiliated Hospital of Zhejiang University, School of Medicine, Hangzhou 310009, People's Republic of China.

Received: 24 June 2021 Accepted: 24 November 2021 Published online: 11 December 2021

\section{References}

1. Bassetti M, Vena A, Castaldo N, Righi E, Peghin M. New antibiotics for ventilator-associated pneumonia. Curr Opin Infect Dis. 2018;31(2):177-86.

2. Rodriguez-Bano J, Gutierrez-Gutierrez B, Machuca I, Pascual A. Treatment of infections caused by extended-spectrum-beta-lactamase-, AmpC-, and carbapenemase-producing Enterobacteriaceae. Clin Microbiol Rev. 2018;31(2).

3. Du X, He F, Shi Q, Zhao F, Xu J, Fu Y, et al. The rapid emergence of tigecycline resistance in blaKPC-2 harboring Klebsiella pneumoniae, as mediated in vivo by mutation in tetA during tigecycline treatment. Front Microbiol. 2018;9:648.

4. Veleba M, De Majumdar S, Hornsey M, Woodford N, Schneiders T. Genetic characterization of tigecycline resistance in clinical isolates of Enterobacter cloacae and Enterobacter aerogenes. J Antimicrob Chemother. 2013;68(5):1011-8.

5. Yoon EJ, Oh Y, Jeong SH. Development of tigecycline resistance in carbapenemase-producing Klebsiella pneumoniae sequence type 147 via AcrAB overproduction mediated by replacement of the ramA promoter. Ann Lab Med. 2020;40(1):15-20.

6. Sun Y, Cai Y, Liu X, Bai N, Liang B, Wang R. The emergence of clinical resistance to tigecycline. Int J Antimicrob Agents. 2013;41(2):110-6.

7. Gasparrini AJ, Markley JL, Kumar H, Wang B, Fang L, Irum S, et al. Tetracycline-inactivating enzymes from environmental, human commensal, and pathogenic bacteria cause broad-spectrum tetracycline resistance. Commun Biol. 2020;3(1):241.

8. He T, Wang R, Liu D, Walsh TR, Zhang R, LV Y, et al. Emergence of plasmidmediated high-level tigecycline resistance genes in animals and humans. Nat Microbiol. 2019;4(9):1450-6.

9. Liu DJ, Zhai WS, Song HW, Fu YL, Schwarz S, He T, et al. Identification of the nove tigecycline resistance gene tet(X6) and its variants in Myroides, Acinetobacter and Proteus of food animal origin. J Antimicrob Chemoth. 2020;75(6):1428-31.

10. Sun J, Chen C, Cui CY, Zhang Y, Liu X, Cui ZH, et al. Plasmid-encoded tet $(X)$ genes that confer high-level tigecycline resistance in Escherichia coli. Nat Microbiol. 2019;4(9):1457-64

11. Wang LY, Liu DJ, LV Y, Cui LQ, Li Y, Li TM, et al. Novel plasmid-mediated tet $(X 5)$ gene conferring resistance to tigecycline, eravacycline, and omadacycline in a clinical Acinetobacter baumannii isolate. Antimicrob Agents Ch. 2020;64(1)

12. Zheng XR, Zhu JH, Zhang J, Cai P, Sun YH, Chang MX, et al. A novel plasmid-borne tet(X6) variant co-existing with bla(NDM-1) and bla(OXA-58) in a chicken Acinetobacter baumannii isolate. J Antimicrob Chemoth. 2020;75(11):3397-9.

13. Zhang R, Dong N, Zeng Y, Shen Z, Lu J, Liu C, et al. Chromosomal and plasmid-borne tigecycline resistance genes tet $(X 3)$ and tet $(X 4)$ in dairy cows on a Chinese farm. Antimicrob Agents Chemother. 2020;64(11):e00674-20.

14. Laskowski RA, Swindells MB. LigPlot+: multiple ligand-protein interaction diagrams for drug discovery. J Chem Inf Model. 2011:51(10):2778-86.

15. Volkers G, Palm GJ, Weiss MS, Wright GD, Hinrichs W. Structural basis for a new tetracycline resistance mechanism relying on the TetX monooxygenase. FEBS Lett. 2011;585(7):1061-6.

16. Sievers F, Wilm A, Dineen D, Gibson TJ, Karplus K, Li W, et al. Fast, scalable generation of high-quality protein multiple sequence alignments using Clustal Omega. Mol Syst Biol. 2011;7:539.

17. Robert X, Gouet P. Deciphering key features in protein structures with the new ENDscript server. Nucleic Acids Res. 2014;42(Web Server issue):W320-4.

18. Volkers G, Damas JM, Palm GJ, Panjikar S, Soares CM, Hinrichs W. Putative dioxygen-binding sites and recognition of tigecycline and minocycline in 
the tetracycline-degrading monooxygenase TetX. Acta Crystallogr D Biol Crystallogr. 2013;69(Pt 9):1758-67.

19. Walkiewicz K, Benitez Cardenas AS, Sun C, Bacorn C, Saxer G, Shamoo Y. Small changes in enzyme function can lead to surprisingly large fitness effects during adaptive evolution of antibiotic resistance. Proc Natl Acad Sci U S A. 2012;109(52):21408-13.

20. Chopra I, Roberts M. Tetracycline antibiotics: mode of action, applications, molecular biology, and epidemiology of bacterial resistance. Microbiol Mol Biol Rev. 2001;65(2):232-60.

21. Grossman TH. Tetracycline antibiotics and resistance. Cold Spring Harbor Perspect Med. 2016;6(4):a025387.

22. Nguyen F, Starosta AL, Arenz S, Sohmen D, Donhofer A, Wilson DN. Tetracycline antibiotics and resistance mechanisms. Biol Chem. 2014;395(5):559-75.

23. Pankey GA. Tigecycline. J Antimicrob Chemoth. 2005;56(3):470-80.

24. Draper MP, Weir S, Macone A, Donatelli J, Trieber C, Tanaka S, et al. Mechanism of action of the novel aminomethylcycline antibiotic omadacycline. Antimicrob Agents Ch. 2014;58(3):1279-83.

25. Zhanel GG, Cheung D, Adam H, Zelenitsky S, Golden A, Schweizer F, et al. Review of eravacycline, a novel fluorocycline antibacterial agent. Drugs. 2016;76(5):567-88.

26. Cheng Y, Chen Y, Liu Y, Guo Y, Zhou Y, Xiao T, et al. Identification of novel tetracycline resistance gene tet $(X 14)$ and its co-occurrence with tet $(X 2)$ in a tigecycline-resistant and colistin-resistant Empedobacter stercoris. Emerg Microbes Infect. 2020;9(1):1843-52.

27. Nestl BM, Hauer B. Engineering of flexible loops in enzymes. Acs Catal. 2014; 4(9):3201-11.

28. Linkevicius M, Sandegren L, Andersson DI. Potential of tetracycline resistance proteins to evolve tigecycline resistance. Antimicrob Agents Chemother. 2016;60(2):789-96.

29. Zhou Y, Gregor VE, Sun Z, Ayida BK, Winters GC, Murphy D, et al. Structureguided discovery of novel aminoglycoside mimetics as antibacterial translation inhibitors. Antimicrob Agents Chemother. 2005;49(12):4942-9.

30. Woon EC, Zervosen A, Sauvage E, Simmons KJ, Zivec M, Inglis SR, et al. Structure guided development of potent reversibly binding penicillin binding protein inhibitors. ACS Med Chem Lett. 2011;2(3):219-23.

31. CLSI. Performance standards for antimicrobial susceptibility testing. In: Clinical and Laboratory Standards Institute. 30th ed. Wayne, PA; 2020.

32. Wang Q-S, Zhang K-H, Cui Y, Wang Z-J, Pan Q-Y, Liu K, et al. Upgrade of macromolecular crystallography beamline BL17U1 at SSRF. Nuclear Sci Tech. 2018;29(5):68.

33. Kabsch W. Xds. Acta Crystallogr D Biol Crystallogr. 2010;66(Pt 2):125-32.

34. Winter G. xia2: an expert system for macromolecular crystallography data reduction. J Appl Crystallogr. 2010;43(1):186-90.

35. Evans P. Scaling and assessment of data quality. Acta Crystallogr Sec D Biol Crystallogr. 2006;62(1):72-82

36. Adams PD, Afonine PV, Bunkoczi G, Chen VB, Davis IW, Echols N, et al. PHENIX: a comprehensive Python-based system for macromolecular structure solution. Acta Crystallogr D Biol Crystallogr. 2010;66(Pt 2):213-21.

37. Murshudov GN, Skubak P, Lebedev AA, Pannu NS, Steiner RA, Nicholls RA, et al. REFMAC5 for the refinement of macromolecular crystal structures. Acta Crystallogr D Biol Crystallogr. 2011;67(Pt 4):355-67.

38. Emsley P, Cowtan K. Coot: model-building tools for molecular graphics. Acta Crystallogr D Biol Crystallogr. 2004;60(Pt 12 Pt 1):2126-32.

39. DeLano WL: The PyMOL molecular graphics system. 2002. http.//uww.pymol.org Accessed 1 May 2021

40. Yang HY, Yang SN, Kong JL, Dong AC, Yu SN. Obtaining information about protein secondary structures in aqueous solution using Fourier transform IR spectroscopy. Nat Protoc. 2015;10(3):382-96.

41. Rabbani G, Baig MH, Lee EJ, Cho WK, Ma JY, Choi I. Biophysical study on the interaction between eperisone hydrochloride and human serum albumin using spectroscopic, calorimetric, and molecular docking analyses. Mol Pharmaceut. 2017:14(5):1656-65.

42. Micsonai A, Wien F, Bulyaki E, Kun J, Moussong E, Lee YH, et al. BeStSel: a web server for accurate protein secondary structure prediction and fold recognition from the circular dichroism spectra. Nucleic Acids Res. 2018;46(W1):W315-22.

43. Micsonai A, Wien F, Kernya L, Lee YH, Goto Y, Refregiers M, et al. Accurate secondary structure prediction and fold recognition for circular dichroism spectroscopy. Proc Natl Acad Sci U S A. 2015;112(24):E3095-103.

\section{Publisher's Note}

Springer Nature remains neutral with regard to jurisdictional claims in published maps and institutional affiliations.

Ready to submit your research? Choose BMC and benefit from:

- fast, convenient online submission

- thorough peer review by experienced researchers in your field

- rapid publication on acceptance

- support for research data, including large and complex data types

- gold Open Access which fosters wider collaboration and increased citations

- maximum visibility for your research: over $100 \mathrm{M}$ website views per year

At BMC, research is always in progress.

Learn more biomedcentral.com/submissions 